Document downloaded from:

http://hdl.handle.net/10251/48657

This paper must be cited as:

Canto, B.; Cardona, S. C.; Coll, C.; et ál..(2012). Dynamic optimization of a gas-liquid reactor. Journal of Mathematical Chemistry. 50(2):381-393. doi:10.1007/s10910-011-99411.

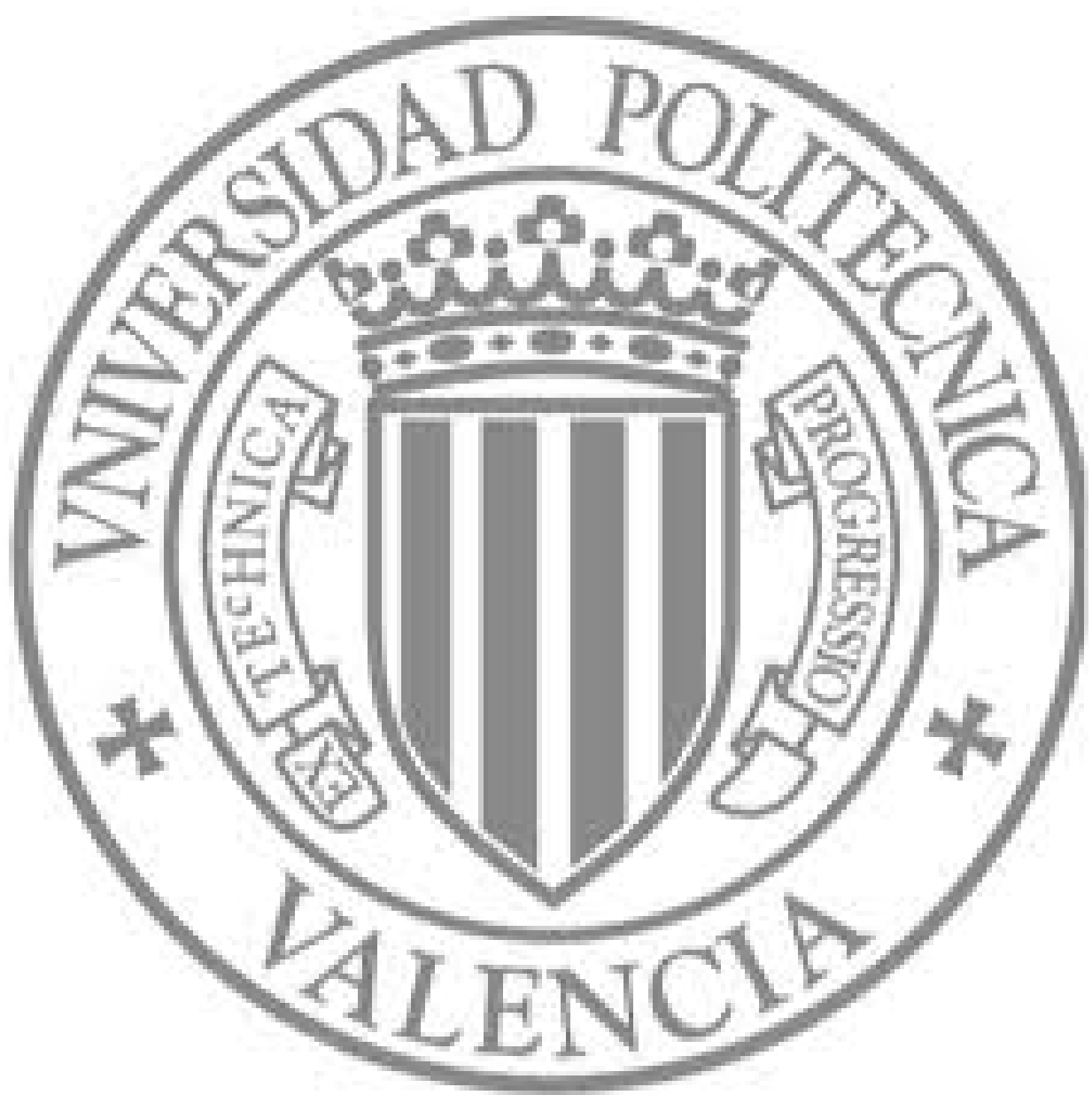

The final publication is available at

http://dx.doi.org/10.1007/s10910-011-9941-1

Copyright Springer Verlag (Germany) 


\title{
Dynamic optimization of a gas-liquid reactor
}

\author{
B.Cantó - S.C. Cardona - C.Coll • J. \\ Navarro-Laboulais • E. Sánchez
}

the date of receipt and acceptance should be inserted later

\begin{abstract}
A dynamic gas-liquid transfer model without chemical reaction based on unsteady film theory is considered. In this case, the mathematical model presented for gas-liquid mass-transfer processes is based on mass balances of the transferred substance in both phases. The identificability property of this model is studied in order to confirm the possible identifiable parameters of the model from a given set of experimental data. For that, a different modeled of the system is given. A procedure for the identification is proposed. On the other hand, the aim of this work is to solve the quadratic optimal control problem, using an explicit representation of the model. The problem includes some results on controllability, observability and stability criteria and the relation between these properties and the parameters of the model. Using the optimal control problem we study the stability of the system and show how the choice of the weighting matrices can improve the behavior of the system but with an increase of the energy control cost.
\end{abstract}

Keywords Continuous system · Structural properties $\cdot$ Stability · Optimal control $\cdot$ Riccatti equation

Mathematics Subject Classification (2000) $34 \cdot 93$

B.Cantó

Institut de Matemàtica Multidisciplinar, Universitat Politècnica de València, Camino de Vera s/n, 46022, Valencia, España

E-mail: bcanto@mat.upv.es

S.C. Cardona · J. Navarro-Laboulais

Departament d'Enginyeria Química i Nuclear, Universitat Politècnica de València, Camino de Vera s/n, 46022, Valencia, España

C.Coll · E. Sánchez

Institut de Matemàtica Multidisciplinar, Universitat Politècnica de València, Camino de Vera s/n, 46022, Valencia, España 


\section{Introduction}

Batch processing is having increasingly importance in chemical and biochemical applications. Mathematical models are used to simulate the behavior of these processes. These models can be constructed in different ways and differ greatly in complexity but they have in common that they can be used to predict the behavior of the initial system. Usually, the model is constructed using some of the properties of a system by tinkering with adjustable parameters. In particular, in the case of bubble column reactors, widely used in chemical, petrochemical, biochemical and metallurgical industries, the design and scale-up of bubble columns are difficult because of the complexity of the gas and liquid flow patterns coupled with mass transfer and chemical reactions. Key factors such as gas hold-up, $\epsilon$, volumetric mass transfer coefficient, $k_{L} a$, specific interfacial area, $a$, bubble size, $r_{32}$, and kinetic rate constants, $k_{n}$, and how those parameters are related is fundamental for the proper design and the operational control of gas-liquid reactors. On the other hand, the absence of moving parts, their low operating and maintenance costs and the excellent mass and heat transfer rates explain the large number of applications developed with this kind of reactor against the others [7], [8]. However, the pitfall in the use of a theoretical model is to know in advance if its parameters can be determined uniquely. After solving the identifiability problem one obtain a model designed to represent theoretical concepts, and to serve as bases for devising quantitative relations and for predicting properties. In the last years, a great variety of works have been developed for the identifiability of this kind of chemical models [5] and [12].

On the other hand, model-based dynamic optimization is used to determine optimal grade changeover policies. The objective of optimal control process is to determine the control signals that will cause a process to satisfy the physical constraints and at the same time minimize (or maximize) some performance criterion. Optimal control problems can be formulated in terms of a cost functional that is a function of state and control variables. This problem is usually described by ordinary or partial differential equation systems describing the paths of the control variables that minimize the cost functional. However, optimal transition policies should not be implemented without feedback control, that is, the optimal control can be expressed in feedback form, where the "feedback gains" satisfy a matrix differential equation of Riccati type. Obtaining the solution of optimal control problems is an important aspect in the analysis of the model. This topic has been studied by several authors. In particular, this subject has been studied in [1] that has a special focus on problems that arise in chemical engineering. In the infinite-time case by using the theory of controllability, the asymptotic behavior of the controlled system can be studied and a rather complete solution to the problem is available, see [9] for more details.

In this paper, we shall concentrate on the special structure that appears in a dynamic gas-liquid reactor, and it is concerned with a study of the optimal feedback control problem for this reactor. It is clear that, see [9], optimal 
control theory is intimately connected no only to system structural properties of controllability and observability but also to the property that relates to the system response to inputs or disturbances, the stability. If one want to study the optimal control problem, the system must be controllable and observable. The goal in optimal control theory is to transfer a system from an arbitrary initial state to the origin while minimizing some performance measure. On the other hand, it is important to note that although practical optimal control systems that minimize quadratic performance indexes are almost always asymptotically stable. In the paper, the theory is currently being completed in order to show the relation of the theory of controllability and observability to the infinite-time quadratic cost problem.

The paper is organized as follows. Section 2 presents the mathematical model of a semi-batch GL reactor and the mathematical ground of our work. Section 3 gives a modification of the initial mathematical model based in a finite difference scheme, the corresponding identifiability analysis and a detailed explanation of the steps of our algorithm for the identification. In Section 4 we study the structural properties and the stability of the model. Finally, in Section 5 we shall set out our problem in terms of optimal control.

\section{Mathematical model of a semi-batch GL reactor}

The classical description of mass transfer processes in gas-liquid reactors makes use of two spatial-temporal scales which one refers to the physical masstransfer at the gas-liquid interface level, i.e. the microscopic model, and the other time scale is referred to the modelling of the reactor configuration which considers the mixing processes and the chemicals distribution in the whole volume of the reactor, i.e. the macroscopic model. We consider a semi-batch GL reactors, see figure 1, where a gas mixture containing a soluble gas and an inert one, is bubble through the liquid.

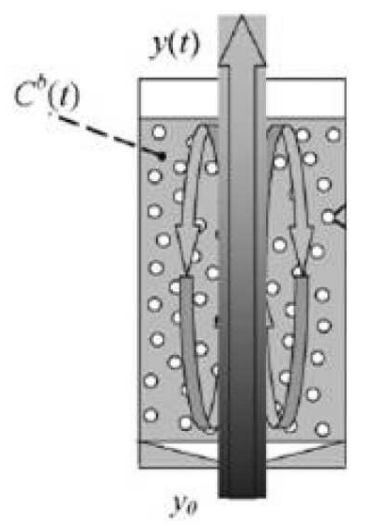

Fig. 1 Representation of the model. 
In this model we consider the following assumptions:

a) The liquid and the gas bulk are completely mixed.

b) The equation of state of ideal gases and the Henrys law could be applied.

c) Initially there is no dissolved gas in the liquid phase but the inert gas flowing through the liquid column.

d) The macroscopic observable state variables are the mole fraction in the gas phase and the molar concentration in the bulk liquid phase.

e) The is a rigid liquid film of thickness between the gas and the liquid bulk where the mass transport is by molecular diffusion. The gas and liquid concentrations at the interface are always in equilibrium.

f) There is not gas-film resistance for mass-transfer.

g) The microscopic state variables are given by the molar concentration inside the liquid film, which are not experimentally accessible.

Moreover, in our case, the temperature and pressure are constant. Thus, there are not chemical reactions.

The mass balance for the gas phase is given as follows.

$$
\frac{d y(t)}{d t}=\frac{R T}{P V} \frac{1-\epsilon}{\epsilon} F_{i}\left(\frac{y_{0}}{1-y_{0}} U(t)-\frac{y(t)}{1-y(t)}\right)+\frac{R T}{P} \frac{1-\epsilon}{\epsilon} a D\left(\frac{\partial C(z, t)}{\partial z}\right)_{z=0}
$$

with the initial condition $y(0)=0$, where $y_{0}$ and $y(t)$ are the mole fraction of a soluble gas in the gas phase at the reactor inlet and outlet, respectively, $R$ in the universal gas constant $\left(8.13 J K-1 \mathrm{~mol}^{-1}\right), T$ in the reactor temperature $(K), P$ is the pressure (atm), $V$ is the volume of the liquid phase in the reactor $\left(\mathrm{m}^{3}\right), \epsilon$ is the reactor gas hold-up, $F_{i}$ is the inert gas molar flow rate $\left(\mathrm{mol} \mathrm{s} s^{-1}\right)$, $a$ is the interfacial specific area $\left(m^{-1}\right)$ and $D$ is the diffusion coefficient of the gas in the liquid film $\left(m^{2} s^{-1}\right) . C(t)$ is the molar concentration in the liquid bulk $\left(\mathrm{mol} \mathrm{m}^{-3}\right)$ and $U(t)$ is the unitary step function defined as

$$
U(t)= \begin{cases}0 & t \leq 0 \\ 1 & t>0\end{cases}
$$

We introduce this function since the system is initially at steady state $(y(0)=$ 0 ) and it is perturbed by a step of gas concentration at the inlet of the bubble column.

The unsteady mass balance in the liquid bulk is given by

$$
\frac{d C^{b}(t)}{d t}=-a D\left(\frac{\partial C(z, t)}{\partial z}\right)_{z=\delta}
$$

with the initial condition $C^{b}(0)=0$. Finally, at the quiescent liquid film the mass transport is described by Fick's second law with unstationary boundary conditions

$$
\frac{\partial C(z, t)}{\partial z}=D \frac{\partial^{2} C(z, t)}{\partial z^{2}}, \forall z \in[0, \delta]
$$


with the initial boundary conditions:

$$
\begin{aligned}
& t=0 \quad C(z, 0)=0 \\
& t>0 \quad C(0, t)=\frac{P}{H} y(t) \\
& t>0 \quad C(\delta, t)=C^{b}(t)
\end{aligned}
$$

being $\delta$ the quiescent liquid film thickness $(m)$ and $H$ the Henry constant $\left(\operatorname{atm} L \mathrm{~mol}^{-1}\right)$.

The above model is a complex system which analytical solution cannot be solved by conventional techniques. In simplifying the problem it is important to focus on describing the necessary steps to obtain associated system taking account the main reason why the system exists or is being built. For example, in our model we only have considered a simplification of the system to reach a semi-numerical solution. The simplification is based on the transformation of the model using a finite differences scheme. The process is given in the next section.

Now, in this section we remind some mathematical background that we use along this work.

The problem of the structural identifiability of the model consists of the determination of all parameter sets which give the same input-output structure. A characterization of structural identifiability is given in [2]. Thus, a continuous-time system is structurally identifiable if and only if, for almost any two candidates parameter vector values $\mathbf{p}, \mathbf{q} \in \mathcal{P}, i o(\mathbf{p})=i o(\mathbf{q})$ implies $\mathbf{p}=\mathbf{q}$, where $i o(\cdot)$ denotes the input-output behaviour of the system.

Thus structural identifiability analysis is an important step in the modeling process and it is necessary theoretical prerequisites to experiment design and system identification, that is, to estimate the unknown parameters of the model using experimental data (more information in [3] and [4]).

To determine the input-output behavior (io) of the model we can use the Markov parameters associated to the continuous-time system.

Optimal control theory is intimately connected to system structural properties of controllability and observability and to stability. To study the optimal control problem, the system must be controllable and observable. For that we study this properties.

A system is controllable if and only if the system states can be changed by changing the system input. That is, a system is controllable, if given two state $x_{0}=x\left(t_{0}\right) \in \mathbb{R}^{n}$ and $x_{f}=x\left(t_{f}\right) \in \mathbb{R}^{n}$, there exists a time $t_{f}$, with $t_{0}<t_{f}$ and a control vector $u(t)$ defined on de interval $\left[t_{0}, t_{f}\right]$ which takes the state vector state from $x_{0}$ to $x_{f}$.

A system with an initial state is observable if and only if the value of the initial state can be determined from the system output that has been observed through the time interval. That is, a system is said to be observable, if there exist a time $t_{f}$, with $t_{0}<t_{f}$ such that given the vectors $u(t)$ and $y(t)$ over the interval $\left[t_{0}, t_{f}\right]$ it is possible to deduce the initial state-vector $x\left(t_{0}\right)$, see [13] for more details. 
In a chemical process, when two compartments are in contact, they can achieve equilibrium points. An equilibrium point of a continuous-time system denoted by $x^{*} \in \mathbb{R}^{n}$, verifies $x^{*}=A(\mathbf{p}) x^{*}$, being $A(\mathbf{p})$ the system matrix of model. An equilibrium point $x^{*}$ is said to be asymptotically stable if every trajectory starting in a neighborhood of it is around the $x^{*}$ and converges on $x^{*}$. The system is asymptotically stable if all the roots of the characteristic polynomial $|s I-A(\mathbf{p})|$ have negative real part.

One tool used to check if the system is asymptotically stable is the RouthHurwitz stability criterion. Remind that if $a_{n}(s) \in \mathbb{R}[s]$ is a polynomial of order $n$ with real coefficients, and

$$
a_{n}(s)=a_{0} s^{n}+a_{1} s^{n-1}+\ldots+a_{n-1} s+a_{n}
$$

then the Routh array is formed by the rows

$$
R(k)=\left[r_{1}^{(k)} r_{2}^{(k)} \cdots r_{n-k}^{(k)}\right]
$$

for $k=1, \ldots, n+1$. The first two rows are determined by the coefficients of $a_{n}(s)$

$$
\left[\begin{array}{l}
R^{(1)} \\
R^{(2)}
\end{array}\right]=\left[\begin{array}{llll}
a_{0} & a_{2} & a_{4} & \ldots \\
a_{1} & a_{3} & a_{5} & \ldots
\end{array}\right]
$$

Given rows $k-1$ and $k$, the $k+1$-th row, $R^{(k+1)}$ is obtained by

$$
\left[\frac{r_{1}^{(k)} r_{2}^{(k-1)}-r_{1}^{(k-1)} r_{2}^{(k)}}{r_{1}^{(k)}} \frac{r_{1}^{(k)} r_{3}^{(k-1)}-r_{1}^{(k-1)} r_{3}^{(k)}}{r_{1}^{(k)}} \cdots\right]
$$

The system is asymptotically stable if all the coefficients of $a_{n}(s)$ are positive and all there is not any sign changes in the first column of the Routh array.

\section{Identifiability Analysis}

After the film thickness $\delta$ is divided in $N$ equal intervals, with the application of the second-order forward, backward and central differences scheme for the flux term, the following ODE system is obtained from the previous PDE system given in (1-2-3)

$$
\begin{aligned}
\dot{x}_{1} & =\gamma p_{1} U(t)-\alpha K_{2} x_{1}-N \beta \frac{p_{1} p_{3}}{p_{2} p_{4}} p_{5}\left(3 x_{1}-4 x_{2}+x_{3}\right)-\alpha p_{1} \frac{x_{1}^{2}}{K_{2}-x_{1}} \\
\dot{x}_{i} & =N^{2} \frac{p_{3}}{p_{4}^{2}}\left(x_{i-1}-2 x_{i}+x_{i+1}\right) \quad i=2, \ldots, N \\
\dot{x}_{N+1} & =-\frac{N}{2} \frac{p_{3}}{p_{4}} p_{5}\left(x_{N-1}-4 x_{N}+3 x_{N+1}\right) \quad i=2, \ldots, N
\end{aligned}
$$


where the parameters and the constants of the model are defined as

$$
\begin{aligned}
p_{1} & =\frac{1-\epsilon}{\epsilon} ; p_{2}=H ; p_{3}=D ; p_{4}=\delta ; p_{5}=a ; \\
K_{1} & =\frac{R T}{P V} \frac{F_{i}}{y_{0}} ; K_{2}=y_{0}^{-1} ; K_{3}=\frac{1}{2} R T ; \\
\alpha & =\frac{K_{1}}{K_{2}} ; \beta=K_{3} ; \gamma=\frac{K_{1}}{K_{2}-1}
\end{aligned}
$$

All the initial conditions of the equations (4) are set to zero, $x_{i}(0)=0 \forall i$. Note that $x_{1}$ is the normalized mole fraction at the gas phase outlet and $x_{N+1}$ the normalized concentration of the soluble gas in the liquid bulk. They are the two states variables of this system of ordinary differential equations which give the macroscopic observable magnitudes of our problem. In summary, $x_{1}=$ $y(t) / y_{0}, C^{*}=y_{0} P / H$ and $x_{i}=C_{i} / C^{*}$ for $i=2, \ldots, N+1$.

Considering the simplest representation of equations (4) with $N=3$, the model can be modeled using the following linear differential equation

$$
\begin{aligned}
\dot{x}(t) & =A(\mathbf{p}) x(t)+B(\mathbf{p}) u(t) \\
y(t) & =C x(t)
\end{aligned}
$$

where the state matrix $A(\mathbf{p})$, the perturbation matrix $B(\mathbf{p})$ and the observation matrix $C$ are given by

$$
\begin{aligned}
& A(\mathbf{p})=\left(\begin{array}{rrrr}
\alpha q_{1}-9 \beta \frac{q_{1} q_{3} q_{4}}{q_{2}} & 12 \beta \frac{q_{1} q_{3} q_{4}}{q_{2}} & -3 \beta \frac{q_{1} q_{3} q_{4}}{q_{2}} & 0 \\
9 q_{3} & -18 q_{3} & 9 q_{3} & 0 \\
0 & 9 q_{3} & -18 q_{3} & 9 q_{3} \\
0 & -\frac{3}{2} q_{3} q_{4} & 6 q_{3} q_{4} & -\frac{9}{2} q_{3} q_{4}
\end{array}\right) \\
& B(\mathbf{p})=\left(\begin{array}{llll}
\gamma q_{1} & 0 & 0 & 0
\end{array}\right)^{T}, C=\left(\begin{array}{llll}
1 & 0 & 0 & 0 \\
0 & 0 & 0 & 1
\end{array}\right)
\end{aligned}
$$

and the parameter vector $\mathbf{p}$, which belongs to a subset of admissible parameters $\mathcal{P} \subseteq \mathbb{R}^{r}$, is given by the new parameters:

$$
q_{1}=\frac{1-\epsilon}{\epsilon} ; q_{2}=H ; q_{3}=\frac{D}{\delta^{2}} ; q_{4}=\delta a
$$

The advantage of the above approximation is that it reduces the complexity of the system, passing from a partial differential equation to a differential equation and thus speeds up numerical simulations greatly and simplify the analysis of the identifiability property. For that, we use the solution of this system given by

$$
y(t)=C e^{A(\mathbf{p}) t} x(0)+C \int_{0}^{t} e^{A(\mathbf{p})(t-\tau)} B(\mathbf{p}) u(\tau) d \tau
$$

and the Markov parameters given by

$$
V(j, \mathbf{p})=C A^{j}(\mathbf{p}) B(\mathbf{p}), j \geq 0 .
$$


Now, we study the identificability of the model (5). In [11] we can see that the system (4) is not identifiable. But our model treats the variables in different form, so, we can study the property again.

The parameter identification process for the structured system is followed from the structure of the vectors obtained in (7). We solve the identification problem for the system in the next result.

Proposition. The structured system (5) where $A(\mathbf{p})$ and $B(\mathbf{p})$ are defined by (6) with $\mathbf{p} \in \mathcal{P}$ is structurally identifiable.

Proof. We consider two structured systems defined by (6) with $\mathbf{p}, \mathbf{q} \in \mathcal{P}$ such that they have the same input-output behavior (io)

$$
V(k, \mathbf{p})=V(k, \mathbf{q}), k \geq 0
$$

and we shall prove that $\mathbf{p}=\mathbf{q}$. By the structure of the Markov parameters (7) we have

$$
\begin{aligned}
& V_{1}(1, \mathbf{p})=V_{1}(1, \mathbf{q}) \Rightarrow q_{1}=\bar{q}_{1} \\
& V_{1}(3, \mathbf{p})=V_{1}(3, \mathbf{q}) \Rightarrow q_{2}=\bar{q}_{2}, \\
& V_{1}(2, \mathbf{p})=V_{1}(2, \mathbf{q}) \Rightarrow q_{3} q_{4}=\bar{q}_{3} \bar{q}_{4}, \\
& V_{2}(3, \mathbf{p})=V_{2}(3, \mathbf{q}) \Rightarrow q_{3}^{2} q_{4}=\bar{q}_{3}^{2} \bar{q}_{4},
\end{aligned}
$$

Using the two last equations we obtain $q_{4}=\bar{q}_{4}$ and $q_{3}=\bar{q}_{3}$. Hence, $\mathbf{p}=\mathbf{q}$ and the system is identifiable.

Now, we introduce an algorithm to obtain the parameters of our model, using the Markov parameters technique.

Step 1 Introduce the size of the state vector: $n$. Introduce the matrices $\{V(j)=$ $\left.\left(v(j)_{i}\right)_{i=1, \ldots, n}, j=1, \ldots, n\right\}$ that determine the known input-output behavior of the process. Introduce $\alpha, \beta$ and $\gamma$

Step 2 Obtain $q_{1}=\frac{v(1)_{1}}{\gamma}$

Step 3 Construct $\mathcal{A}=\frac{1}{9 q_{1} \beta}\left(-q_{1} \alpha-\frac{v(2)_{1}}{v(1)_{1}}\right)$

Step 4 Construct $\mathcal{B}=\frac{-2 v(3)_{2}}{27 q_{1} \gamma}$

Step 5 Construct $\mathcal{C}=\frac{v(3)_{1}}{v(1)_{1}}-\left(\frac{v(2)_{1}}{v(1)_{1}}\right)^{2}$

Step 6 Obtain $q_{2}=\frac{108 q_{1} \beta \mathcal{B}}{\mathcal{C}}$

Step 8 Obtain $q_{3}=\frac{\mathcal{B}}{2 q_{2} \mathcal{A}}$

Step 9 Obtain $q_{4}=\frac{\mathcal{B}}{q_{3}^{2}}$

This algorithm has been implemented in MatLab. Since the parameters of the system can be obtained, for simplicity, from now on we ignore the dependence of the coefficient matrices of the system on $\mathbf{p}$, and they shall be denoted by $A$ and $B$. 


\section{Controllability, Observability and Stability criteria}

In this section we check if the system verifies the controllability and observability properties. We give the next result.

Proposition. Consider the system given in (5) with the matrices (6). This system is controllable and observable.

Proof. We consider the matrices given in (6) and construct the rechability matrix $\mathcal{R}=\left[B A B A^{2} B A^{3} B\right]$. Since $\operatorname{rank}(\mathcal{R})=4=n$ being $n$ the size of the system. Then the system (5) is controllable (see [6] for more information). On the other hand, the system is also observable, since the rank of the observability matrix, [6],

$$
\operatorname{rank}\left[\begin{array}{c}
C \\
C A
\end{array}\right]=4=n
$$

is full.

Finally, in our chemical process, to check if the system is asymptotically stable we use the RouthHurwitz stability criterion. This criterion is a procedure widely used to study the stability of continuous- and discrete-time linear systems [10]. This method is able to establish the location of the zeros of a real-coefficient polynomial with respect to the imaginary axis, without actually solving for the zeros. Thus, the Routh-Hurwitz stability test must be applied to the characteristic equation of the system. Let $C_{-}$be the open left half plane $\operatorname{Real}(z)<0$, and $\operatorname{deg}_{-}(P)$ the number of zeros of the characteristic polynomial of the system in $C_{-}$. The system is asymptotically stable if $\operatorname{deg}_{-}(P)=n$, where $n$ is the size of the state matrix. The theorem of Routh-Hurwitz states that $P$ is stable if and only if $H_{k}>0$ for all $k$, where $H_{k}$ is the $k-t h$ subresultant of the polynomials $\sum_{i} p_{2 i} z^{i}$ and $\sum_{i} p_{2 i+1} z^{i}$ being $p_{k}$ the coefficients of $P[14]$.

If we consider our problem and we apply this criteria to the system (5), we obtain that all the polynomial coefficients and $H_{k}$ are positive for all $k$. These results guarantee the asymptotic stability of the system. We summarize the last coments in the the next proposition.

Proposition. Consider the system given in (5) with the matrices (6). This system is asymptotically stable.

\section{Optimal control}

Optimal control deals with the problem of finding a control for a system such that a certain optimality criterion is achieved. A control problem includes a cost functional that is a function of state and control variables. An optimal control is a set of differential equations describing the paths of the control variables that minimize the cost functional. The system dynamic is described by a set of linear differential equations and the cost is described by a quadratic functional. In this case, the solution is provided by the linear-quadratic regulator $(\mathrm{LQR})$. 
We consider the quadratic optimal control problem where the process is an infinite stage process, that is, where $N=\infty$. As $N$ approaches infinity, the optimal control solution becomes a steady-state solution and the time-varying gain matrix $K(k)$ becomes a constant gain matrix written as $K$. To define an optimal control problem we also need a cost function. For $N=\infty$, the cost function subject to the linear time-invariant first-order system (5) is given by

$$
J=\frac{1}{2} \int_{0}^{\infty}\left(x(t)^{T} Q x(t)+u(t)^{t} R u(t)\right) d t
$$

where $x(t)$ is the state, $u(t)$ is the control and the matrices $\mathrm{Q}$ and $\mathrm{R}$ are constant and positive-semidefinite and positive-definite, respectively.

The feedback that minimizes the cost function has the form

$$
u(t)=-K x(t)=-R^{-1} B^{T} P x(t),
$$

where $P$ is the solution of the differential Riccati equation. The differential Riccati equation is given as

$$
P A+A^{T} P-(P B) R^{-1}\left(B^{T} P\right)+Q=0 .
$$

In optimal control matrices $Q$ and $R$ affect directly the values of the system signals $x(t)$ and $u(t)$. It is known that one variable is optimized if the matrix coefficients corresponding to this variable are higher. So, depends the value of the variables, the matrices $Q$ and $R$ are different.

We can interpret the cost criterion as follows. Since $Q$ is positive semidefinite, that is, $x^{T}(t) Q x(t) \geq 0$ and represents the penalty incurred at time $t$ for state trajectories which deviate from 0 . Similarly, since $R$ is positive definite, $u^{T}(t) R u(t)>0$ unless $u(t)=0$ and it represents the control effort at time $t$ in trying to regulate $x(t)$ to 0 . The admissible control requirement ensures that state regulation occurs as $t \rightarrow \infty$. The choice of the weighting matrices $Q$ and $R$ reflects the tradeoff between the requirements of regulating the state to 0 and the expenditure of control energy.

Generally, the matrix $R$ is the identity matrix and the matrix $Q=\left[q_{i}\right]_{i=1}^{n}$ is a diagonal matrix where the relative sizes of $q_{i}$ indicate the relative importance that the designer attaches to $x_{i}(t)$ being away from 0 . The relationship between these matrices is given, for example, as follows:

a) If the matrix $R$ increases, gains decrease and the energy control cost decreases. But with large values of the matrix $R$, the behaviour of the system can worsen.

b) If $R>Q Q$, the contribution of the control $u(t)$ is very large compared with the contribution of the state $x(t)$. That is, the cost decreases but the behaviour of the system worsens.

c) If $Q>>R$, the system response improves but the energy control cost increases. The state vector $x(t)$ will be very controlled, but control signals $u(t)$ can be very large. 
Since our model satisfies the structural properties of controllability and observability and the stability property, then the optimal control problem can be solved. Using the obtained solution, the relationship between the stability of the initial system and the closed loop system can be analyzed. Note that the choice of weighting matrices can improve system performance but with an increase of the energy control cost. These results are then applied to some examples.

\subsection{Numerical results}

Consider the system (5)-(6) with the following values of the parameters [11]: $q_{1}=49, q_{2}=100 \mathrm{~atm} L \mathrm{~mol}^{-1}, q_{3}=50.0000 \mathrm{~s}^{-1}, q_{4}=10^{-3}$.

This system is asymptotically stable and the eigenvalues are

$$
E=[-1350.800696-451.442142-1.958178-0.034606] .
$$

If the weighting matrices are $R=I_{4}$ and $Q=I_{4}$, using Matlab programm, we obtain the following results:

- the time-varying gain matrix $K$ is given by

$$
K=\left[\begin{array}{llll}
0.528512 & 0.003104 & 0.001580 & 0.752653
\end{array}\right]
$$

- the matrix $P$ that solves the differential Riccati equation is given by

$$
P=\left(\begin{array}{cccc}
0.351923 & 0.002280 & 0.001161 & 0.552968 \\
0.002280 & 0.000752 & 0.000376 & 0.004845 \\
0.001161 & 0.000376 & 0.000745 & 0.007710 \\
0.552968 & 0.004845 & 0.007710 & 16.383734
\end{array}\right)
$$

- and the generalized eigenvalues are

$$
E=[-1350.800658-451.441137-2.587221-0.058590] .
$$

Note that if one entry of the matrix $Q$ is changed, this fact influences in the stability of the system. For example, if $Q=\left(\begin{array}{cccc}1 & 0 & 0 & 0 \\ 0 & 1 & 0 & 0 \\ 0 & 0 & 1 & 0 \\ 0 & 0 & 0 & 20\end{array}\right)$, then

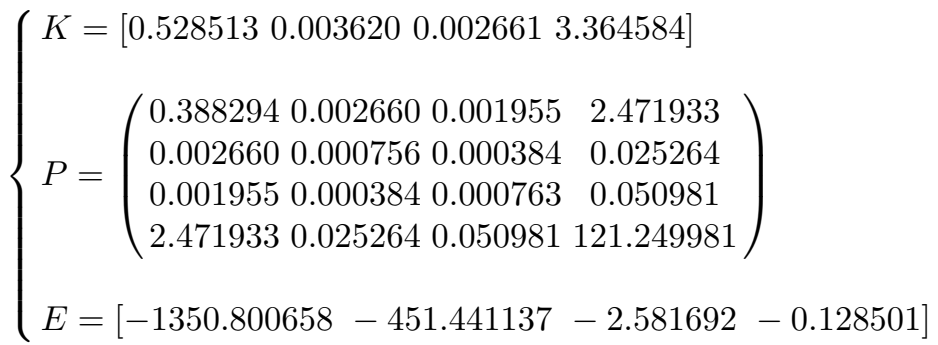


Note that in this case $q_{4}$ has more importance that the rest of the entries of the matrix $Q$. As $Q>R$ the system response improves, that is the stability is better, but the energy control cost increases, since the values of $K$ increase.

$$
\begin{aligned}
& \text { If consider } Q=\left(\begin{array}{llll}
1 & 0 & 0 & 0 \\
0 & 1 & 0 & 0 \\
0 & 0 & 1 & 0 \\
0 & 0 & 0 & 200
\end{array}\right) \text {, then }
\end{aligned}
$$

Since the value of $q_{4}$ is greater that in the before case, the results are more significant.

We summarize the results. If we increase the gain, the pole moves away from the imaginary axis and improves system stability, but the cost of control increases.

\section{Conclusions}

In this paper we study a control design methodology based on optimization applied to a semi-batch GL reactor with unknown parameters. For that, we modelled the semi-batch GL reactor using control theory and we study the identifiability property. In this case we construct an algorithm to obtain the unknown parameters. Moreover we check if the system associated to the model verifies the properties of controllability, observability and stability. These properties are fundamental in the control optimal study.

On the other hand, control design objectives are formulated in terms of a cost criterion. The optimal control law is the one which minimizes the cost criterion. One of the most remarkable results in linear control theory and design is that if the cost criterion is quadratic, and the optimization is over an infinite horizon, the resulting optimal control law has many nice properties, including that of closed loop stability. Thus, we study this property obtaining interesting results.

Acknowledgements This work has been partially supported by PAID-05-10-003-295. 


\section{References}

1. L. Bayón J. M. Grau M. M. Ruiz P. M. SuÁrez, Initial guess of the solution of dynamic optimization of chemical processes, J. Math. Chem. Modelling 48 (2010) $28-37$.

2. A. Ben-Zvi, P.J. McLellan, K.B. McAuley, Ind. Eng. Chem. Res. 42 (2003) 6607-6618.

3. B. Cantó, C. Coll And E. SÁnchez, Structural identifiability of a model of dialysis, Math. Comp. Modelling 50 (2009) 733-737.

4. B. Cantó, C. Coll And E. SÁnchez, Identifiability of a class of discretized linear partial differential algebraic equations, Math. Problems Eng. (2011) 1-12.

5. G. Craciun And C. PAnteA, Identifiability of chemical reaction networks J Math. Chem. 44 (2008) 244-259.

6. L. Dai, Descriptor Control Systems, (Springer-Verlag, New York, 1989)

7. W. D. DeCKWER, Bubble column reactors, John Wiley and Sons, Chichester, 1992.

8. N. Kantarci, F. Borak and K. O. Ulgen, Bubble column reactors, Proc. Biochem. 40(7) (2005) 2263-2283.

9. H. KAWAKERNAAK AND R. Sivan, Linear Optimal Control Systems, Wiley-Interscience, New York, 1972.

10. B. C. Kuo, Automatic Control Systems, 6th ed. Englewood Cliffs, NJ: Prentice-Hall, 1991.

11. J. Navarro-Laboulais, S. C. Cardona, J. I. Torregrosa, A. Abad and F. López, Practical identifiability analysis in dynamic gas-liquid reactors. Optimal experimental design for mass-transfer parameters determination, Comp. and Chem. Eng. 32 (2008) $2382-2394$.

12. J. Navarro-Laboulais,F. López, J. I. Torregrosa, S. C. Cardona and A. Abad , Transient response, model structure and systematic errors in hybrid respirometers: structural identifiabilit analysis based on OUR and DO measurements, J Math. Chem. 44 (4) (2007) 969-990.

13. R.Patel And N. Munro, Multivariable Systen. Theory and Design, Pergamon Press, New York, 1982.

14. K. Sondergeld , A generalization of the Routh-Hurwitz stability criteria and a application to a problem in robust controller design, IEEE Trans. on Automatic Control AC-28 (10) (1983) 965-970. 